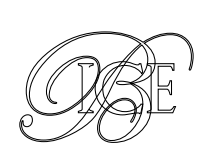

\title{
EL PAPEL DE LA FINANCIACIÓN CLIMÁTICA EN LA CONSECUCIÓN DEL ACUERDO DE PARÍS
}

El Acuerdo de París supone un antes y un después en la manera de afrontar la lucha contra el cambio climático, ya que implica transformar las estrategias de crecimiento económico actuales, dependientes de combustibles fósiles, en estrategias de desarrollo con bajas emisiones y resiliente al clima. Para conseguir este cambio de paradigma es clave contar con una arquitectura financiera climática ágil y flexible. En ella, el papel del sector público es esencial no solo como financiador, sino también como movilizador de la iniciativa privada y garante de unas políticas y regulaciones que permitan realizar una transición adecuada. El grado de involucración del sector privado en la consecución de los objetivos climáticos será determinante a la hora de conseguir su cumplimiento.

Palabras Clave: cambio climático, COP 21, Fondo Verde para el Clima. Clasificación JEL: F30, Q50, Q56.

\section{Introducción}

La Conferencia de las Partes de la Convención Marco de Naciones Unidas sobre el Cambio Climático de 2015, también conocida como COP 21 o Acuerdo de París, supone un profundo cambio en la concepción del grave problema del cambio climático, alcanzando un compromiso mundial sin precedentes para la

\footnotetext{
* Este artículo ha sido elaborado por María Vidales Picazo, Directora Adjunta de Financiación del Desarrollo y Gestión Europea en COFIDES. Técnico Comercial y Economista del Estado. Doctora en Economía.

La autora agradece los comentarios de José Luis Curbelo (Presidente de COFIDES) y de Rodrigo Madrazo (Director General de COFIDES).

Versión de febrero de 2019.

DOI: https://doi.org/10.32796/bice.2019.3109.6776
}

consecución de un desarrollo de bajas emisiones de gases de efecto invernadero y resiliente al clima.

Para alcanzar este ambicioso objetivo, el Acuerdo de París se centra en tres ejes fundamentales: la mitigación del cambio climático (mantener el aumento de la temperatura media mundial por debajo de $2^{\circ} \mathrm{C}$ con respecto a los niveles preindustriales, y proseguir los esfuerzos para limitar ese aumento de la temperatura a $1,5^{\circ} \mathrm{C}$ ), la adaptación (promover la resiliencia al clima) y la financiación (situar los flujos financieros en un nivel compatible con dichos objetivos) (Naciones Unidas, 2015).

La reciente Cumbre del Clima de Katowice (COP 24), en diciembre de 2018, ha permitido desarrollar importantes aspectos del $\square$ 
programa de trabajo del Acuerdo de París, estableciendo unas directrices técnicas y una hoja de ruta (el Paquete Climático de Katowice) para la consecución de los objetivos de la COP 21. Destacan especialmente los acuerdos alcanzados en materia de transparencia (la forma en que los países contabilizarán y reportarán información sobre sus acciones climáticas), los procedimientos establecidos para que el Fondo de Adaptación esté a disposición de los objetivos del Acuerdo de París y el acuerdo de establecer un nuevo objetivo colectivo de financiación climática para 2025 (PNUD, 2018).

La importancia de la lucha contra el cambio climático también está muy presente en los Objetivos de Desarrollo Sostenible (ODS), de tal forma que seis de los diecisiete ODS están relacionados de manera directa o indirecta con la mitigación y adaptación al cambio climático. Se trata de los ODS 6 (Agua limpia y saneamiento), 7 (Energía asequible y no contaminante), 11 (Ciudades y comunidades sostenibles), 13 (Acción por el clima), 14 (Vida submarina) y 15 (Vida de ecosistemas terrestres).

Ahora bien, para conseguir esta ambiciosa hoja de ruta es imprescindible contar con recursos financieros suficientes. Los países desarrollados se han comprometido a contribuir, a través de una amplia variedad de fuentes e instrumentos, con 100.000 millones de dólares anuales a partir de 2020 .

Este artículo analiza las distintas fuentes de financiación climáticas existentes en la actualidad y los retos a los que se enfrentan. Para ello, en el primer apartado se estudiará la arquitectura financiera climática, poniendo el foco tanto en la financiación pública como en la cada vez más importante financiación privada; en segundo lugar, se estudiará en detalle el Fondo Verde para el Clima (GCF, por sus siglas en inglés), al tratarse de una de las principales iniciativas a nivel mundial dedicada al cambio climático; $y$, finalmente, se cerrará el análisis con un apartado de conclusiones.

\section{Arquitectura financiera climática}

La financiación climática se puede definir como aquella que permite alcanzar un desarrollo bajo en carbono y resiliente al cambio climático, lo que constituye una «nueva forma de entender las relaciones económicas, comerciales y de cooperación entre los países» (Montalvo, 2011).

Para llegar a las elevadas cifras de financiación necesarias para estos objetivos, los recursos deben provenir de muy diversas fuentes, siendo las habituales las contribuciones públicas, principalmente de los países desarrollados y de los organismos multilaterales o instituciones financieras de desarrollo. No obstante, el Acuerdo de París abre la puerta al compromiso también de los países en desarrollo (cada uno en función de sus posibilidades), y, principalmente en el marco de los ODS, se reconoce el papel fundamental del sector privado, no solo el filantrópico, sino sobre todo el empresarial.

El Esquema 1 muestra la arquitectura financiera climática a nivel global, donde se puede observar la multiplicidad de actores y la complejidad de la misma.

\subsection{Financiación pública}

Respecto a la financiación pública destinada a la lucha contra el cambio climático, si bien existen muchas contribuciones bilaterales (de países como Reino Unido, Francia, Alemania, España, Dinamarca, Noruega, Estados Unidos, Canadá, Japón o Australia), el grueso de la $\triangleright$ 


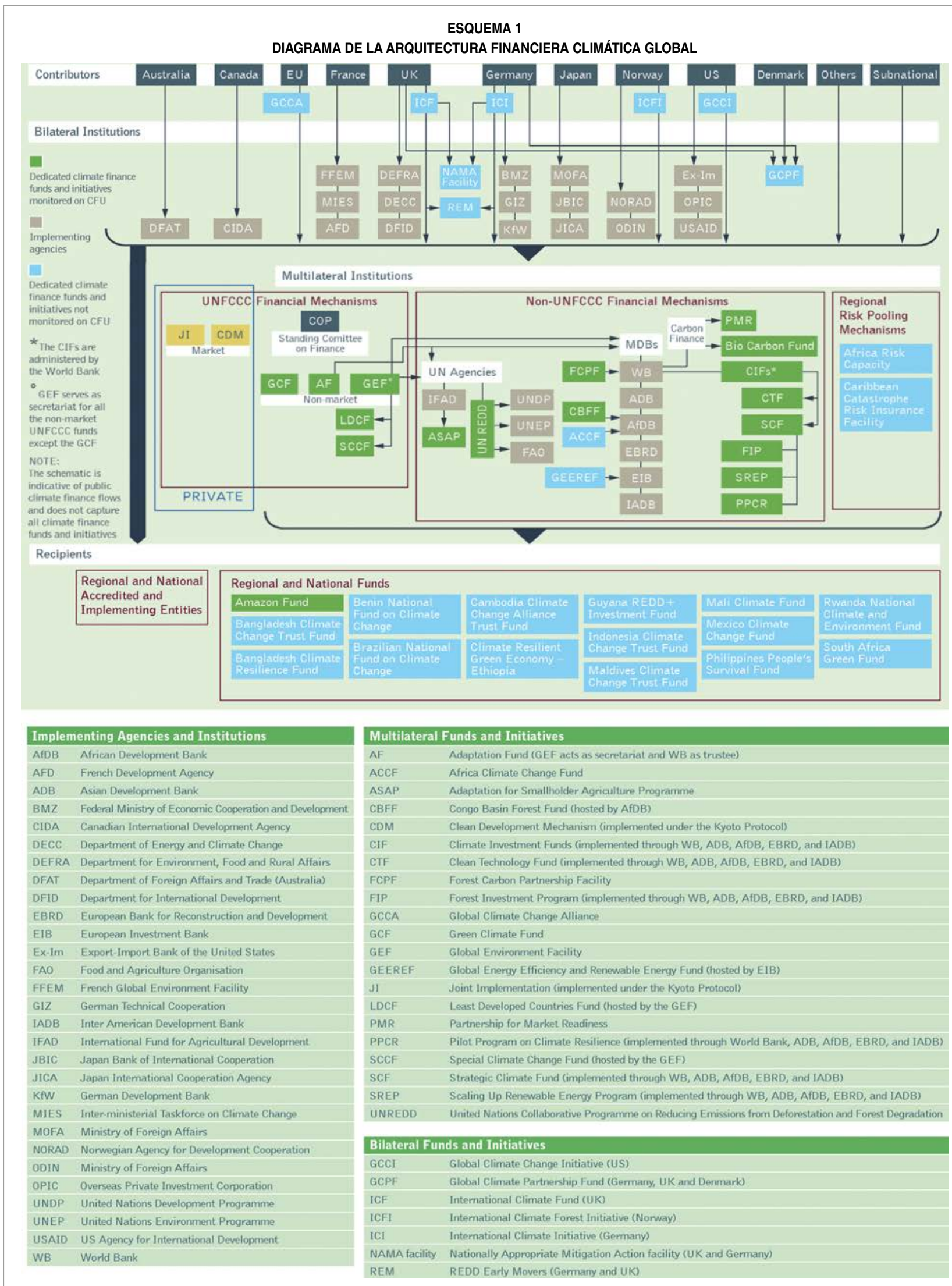

Fuente: Climate Funds Update (CFU, 2017). 
misma se canaliza a través de iniciativas de Naciones Unidas, la Unión Europea o bancos multilaterales de desarrollo.

A nivel de Naciones Unidas, el artículo 10 del Acuerdo de París establece que el Mecanismo Financiero de la Convención Marco sobre el Cambio Climático será el brazo financiero del Acuerdo. Este mecanismo financiero está formado por tres instrumentos: el Fondo para el Medio Ambiente Mundial (FMAM o GEF, por sus siglas en inglés), el Fondo Verde para el Clima y el Fondo de Adaptación (FA).

EI FMAM es una asociación internacional creada en 1991 y formada por 183 países, instituciones internacionales y organizaciones no gubernamentales, que tiene como objetivo proveer de financiación a distintas causas relativas al cambio climático. Desde su constitución ha cofinanciado más de 4.500 proyectos en más de 170 países, proporcionando cerca de 18.000 millones de dólares en donaciones y movilizando más de 93.000 millones (Climate Funds Update, 2017). Además, desde el Secretariado del FMAM se gestionan otros dos fondos fiduciarios: el Fondo Fiduciario Especial sobre el Cambio Climático $(\text { FECC })^{1}$ y el Fondo Fiduciario para los Países Menos Adelantados (FPMA) ${ }^{2}$. España tiene un fuerte compromiso con la lucha contra el cambio climático y desde su ingreso en el FMAM ha comprometido a este fondo más de 191 millones de dólares, ha aportado al FECC más de 12 millones y al FPMA cerca de 2 millones (Ministerio de Economía y Empresa, 2019).

Por su parte, el segundo pilar financiero del Acuerdo de París es el Fondo Verde para el Clima, cuya creación se acordó en la Cumbre

\footnotetext{
Formado por quince donantes, entre los que se encuentra España, financia actividades, programas y medidas climáticas adicionales a las que financia el FMAM.

2 Formado por veinticinco donantes, entre los que se encuentra España, tiene como objetivo contribuir a la preparación e implementación de los Programas Nacionales de Acción para la Adaptación (NAPA) de países en desarrollo.
}

de Cancún a finales de 2010 y, finalmente, se constituyó un año más tarde, en la Cumbre de Durban. Tiene como objetivo financiar proyectos de mitigación y adaptación al cambio climático en países en desarrollo y emergentes. Dada la importancia de este fondo dentro de la arquitectura financiera climática, al tratarse de una de las principales instituciones financieras multilaterales dedicadas al cambio climático, será tratado en profundidad en el apartado 3.

Finalmente, el tercer mecanismo financiero de la Convención Marco sobre el Cambio Climático es el Fondo de Adaptación, el cual nació en 2009 y tiene como objetivo proveer de acceso directo a financiación a entidades nacionales de países en desarrollo encargadas de la implementación de proyectos y programas de adaptación al cambio climático, cumpliendo con los estándares fiduciarios, ambientales, sociales y de género de las agencias de Naciones Unidas. Este fondo obtiene sus recursos tanto de las contribuciones de los países desarrollados como del impuesto sobre la venta de derechos de emisión dentro del Protocolo de Kioto (Climate Funds Update, 2017). Hasta 2018, el Fondo de Adaptación, pese a ser un mecanismo financiero de Naciones Unidas, no estaba directamente vinculado a la consecución de los objetivos del Acuerdo de París; no obstante, en el Paquete Climático de Katowice se ha decidido que, a partir de enero de 2019 , el fondo funcione en el contexto de dicho acuerdo.

Por otra parte, a nivel europeo, la UE, sus Estados miembros y el BEI son conjuntamente el mayor contribuyente a la financiación pública de la lucha contra el cambio climático en los países en desarrollo, con 20.400 millones de euros solo en 2017 (lo que supone cerca de 3.000 millones más que el año anterior). Asimismo, el $20 \%$ del presupuesto total de la UE para el periodo $2014-2020$ se destina $a \triangleright$ 


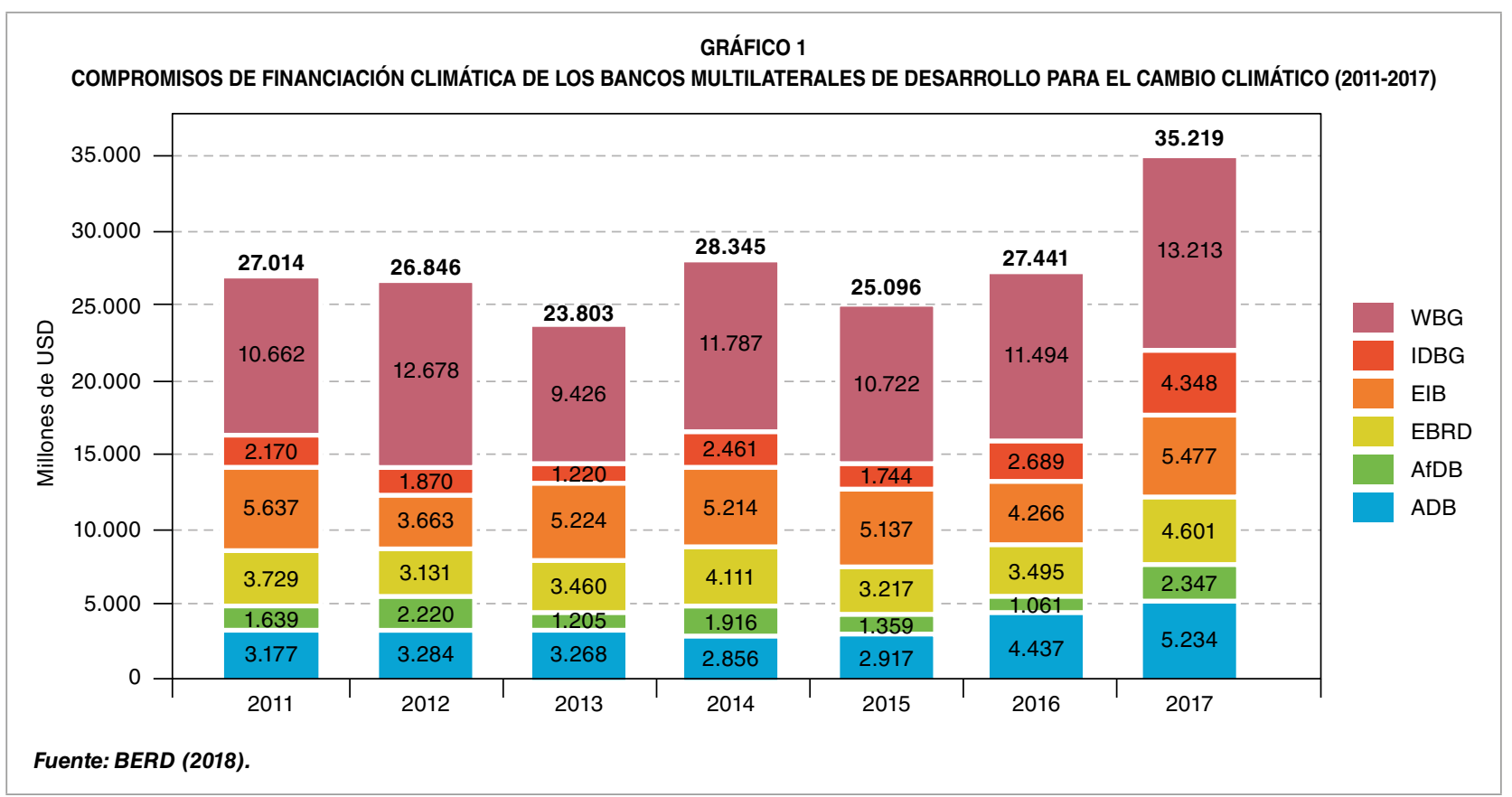

acciones relacionadas con el clima, y la Comisión ha propuesto aumentar esta proporción al menos un $25 \%$ para el nuevo Marco Financiero Plurianual 2021-2027, actualmente en negociación (Comisión Europea, 2019). Además, en marzo de 2018 la Comisión Europea lanzó el Plan de Acción para una economía más ecológica y limpia, que supone una hoja de ruta para que el sistema financiero respalde la estrategia de la UE respecto al cambio climático y el desarrollo sostenible (integrando la sostenibilidad en los requisitos prudenciales, creando etiquetas para productos financieros «verdes", aumentando la transparencia en materia climática en los informes corporativos, entre otras cuestiones) (Comisión Europea, 2018). Asimismo, la UE incorpora el objetivo climático en sus nuevas iniciativas dentro de la Acción Exterior, como es el caso del recientemente creado Plan Europeo de Inversión Exterior o el Programa Invest EU, todavía en negociación.

Por otro lado, como se puede observar en el Esquema 1, además de la financiación en el marco de Naciones Unidas y de la UE, otro eje importante de financiación climática son los seis principales bancos multilaterales de desarrollo $^{3}$. De esta forma, en el año 2017 se alcanzaron los 35.200 millones de dólares aportados por estas seis instituciones, lo que representa casi un 30\% más que el año anterior y permite alcanzar una cifra récord (Gráfico 1). El 79\% de estos recursos se destinaron a proyectos de mitigación del cambio climático y el $21 \%$ restante a adaptación. El principal instrumento financiero utilizado fue el préstamo, pero no fue el único, ya que el $10 \%$ de la financiación de estos bancos de desarrollo multilaterales en 2017 se otorgó en forma de donaciones, capital y garantías, permitiendo movilizar la iniciativa privada (BERD, 2018).

Por su parte, las quince Instituciones Europeas de Financiación del Desarrollo, agrupadas bajo la asociación $\mathrm{EDFI}^{4}$ (por sus siglas $\triangleright$

3 Grupo Banco Mundial (BM), Grupo Banco Interamericano de Desarrollo (BID), Banco Europeo de Inversiones (BEI), Banco Europeo de Reconstrucción y Desarrollo (BERD), Banco Africano de Desarrollo (BAfD) y Banco Asiático de Desarrollo (BAD).

4 La institución financiera de desarrollo española miembro de EDFI es la Compañía Española de Financiación del Desarrollo (COFIDES). 
en inglés), también se muestran activas en la consecución de los objetivos climáticos a nivel mundial. La cartera consolidada de EDFI en proyectos climáticos internacionales superó en 2017 los 8.000 millones de euros, principalmente concentrada en los sectores de energía solar, eólica e hídrica (EDFI, 2017).

\subsection{Financiación privada}

A pesar de la creciente importancia de la financiación pública, para conseguir alcanzar el objetivo de movilización de 100.000 millones de dólares anuales, por todos es conocido que los recursos públicos no son suficientes, por lo que el papel del sector privado se vuelve crucial, y, al mismo tiempo, el papel del sector público se desdobla: como financiador y como «catalizador».

En efecto, el sector público (entendido este en sentido amplio, no solo los recursos cedidos por los Gobiernos, sino también a través de bancos multilaterales e instituciones financieras de desarrollo) debe servir también para movilizar la financiación privada, a través de la mitigación del riesgo, la asistencia técnica y el desarrollo de capacidades. Esto es lo que en la jerga del mundo de la cooperación y en el marco no solo de la financiación climática, sino de la financiación de los ODS, se ha venido a denominar «from billions to trillions». Es decir, que los miles de millones (billions) de recursos públicos aportados no solo sirvan como financiación directa de acciones climáticas, sino también para movilizar la iniciativa privada hasta conseguir los billones de dólares (trillions) necesarios para alcanzar los objetivos del Acuerdo de París (Banco Mundial, 2015).

Por su parte, la participación del sector privado en la lucha contra el cambio climático no debe entenderse solo como una cuestión financiera, sino bajo un prisma mucho mayor, ya que el sector privado puede y debe tener un papel clave a la hora de conseguir una economía neutra en carbono ${ }^{5}$ : incorporando en sus estrategias la cuestión del cambio climático, adaptando su forma de producir y proveer de servicios a través de soluciones de bajas emisiones y con bajo consumo de combustibles fósiles (Burchardt et al., 2018). Con estas medidas, el sector privado no solo estará contribuyendo a la sostenibilidad del planeta, sino que se beneficiará también a nivel empresarial, al favorecer que las compañías participantes sean más competitivas en el mercado.

Del mismo modo, el papel del sector público en la consecución de los objetivos climáticos trasciende la esfera financiera, y es especialmente importante su participación en el desarrollo de políticas públicas de I+D+i, invirtiendo en infraestructuras limpias y proveyendo de formación y capacitación que faciliten la reconversión de determinados sectores. Asimismo, también resulta clave tener un marco regulatorio estable que reduzca la incertidumbre y estimule el cambio. En este nuevo escenario, el sector financiero en los países desarrollados debe estar para ayudar a conseguir esa «transición justa» (Fabius, 2019).

\section{EI Fondo Verde para el Clima}

Como se ha indicado anteriormente, para alcanzar las cifras tan abrumadoras de financiación son necesarias iniciativas a nivel mundial. Todas ellas son importantes, si bien en este artículo se va a poner el énfasis en el $\square$

5 Entendida como el equilibrio entre las emisiones y las absorciones de gases de efecto invernadero. 
Fondo Verde para el Clima, por ser la principal institución financiera multilateral que busca contribuir a la consecución de los objetivos en materia de lucha contra el cambio climático, a través de instrumentos financieros públicos que permitan catalizar la inversión privada, multiplicando el efecto de la financiación inicial. De esta manera, el Fondo Verde, a través de la ventanilla para el sector privado (Private Sector Facility), pretende movilizar recursos procedentes de fondos de pensiones, compañías de seguros, corporaciones, intermediarios financieros locales y regionales y el propio mercado de capitales.

\section{Objetivos}

El objetivo del fondo es promover un cambio de paradigma hacia un desarrollo de bajas emisiones y resiliente al cambio climático. Para ello, pone a disposición una diversidad de instrumentos financieros que pretenden movilizar los recursos suficientes para financiar proyectos, tanto públicos como privados, de mitigación y adaptación al cambio climático (Tabla 1) en países en desarrollo y emergentes ${ }^{6}$. Los instrumentos financieros que pone a disposición el Fondo Verde para el Clima son: donaciones, préstamos concesionales, capital y garantías (GCF, 2018a).

\section{Cartera actual}

El Fondo Verde para el Clima comenzó a financiar proyectos en 2015 y en estos cuatro años ha comprometido 4.600 millones de dólares en 93 proyectos que beneficiarán a más de 270 millones de personas. En enero de 2019, el $39 \%$ de esos proyectos eran de mitigación, el $25 \%$, de adaptación y el $36 \%$ restante eran proyectos transversales. Se trata principalmente de proyectos públicos $(60 \%)$, si bien se han empezado a aprobar proyectos privados, sobre todo contribuciones a fondos de inversión $D$

6 Actualmente son 154 los países elegibles, aquellos firmantes de la Conferencia de las Partes (COP) que figuran en el Non-Annex 1 de Naciones Unidas: https://unfccc.int/process/parties-non-party-stakeholders/partiesconvention-and-observer-states

TABLA 1

TIPOLOGÍA DE PROYECTOS DE MITIGACIÓN Y ADAPTACIÓN AL CAMBIO CLIMÁTICO

\begin{tabular}{|c|c|}
\hline Mitigación & Adaptación \\
\hline $\begin{array}{l}\text { Acciones encaminadas a reducir la emisión de gases de efecto } \\
\text { invernadero o a aumentar las capturas (sumideros) de } \mathrm{CO}_{2}\end{array}$ & $\begin{array}{l}\text { Acciones encaminadas a promover la resiliencia tanto de las } \\
\text { personas como del entorno natural a los efectos del cambio } \\
\text { climático (reales o previstos) }\end{array}$ \\
\hline \multicolumn{2}{|c|}{ Tipología de proyectos } \\
\hline $\begin{array}{l}\text { Producción de electricidad a través de energías renovables en } \\
\text { vez de combustibles fósiles }\end{array}$ & $\begin{array}{l}\text { Aumento de los medios de subsistencia de las personas, } \\
\text { comunidades y regiones más vulnerables al clima }\end{array}$ \\
\hline Transporte de bajas emisiones & Infraestructura resistente a las amenazas del cambio climático \\
\hline Reducción de emisiones en edificios e industrias & Seguridad hídrica y alimentaria ante desastres naturales \\
\hline $\begin{array}{l}\text { Reducción de emisiones derivadas del uso de la tierra, la } \\
\text { deforestación y la degradación de los bosques }\end{array}$ & $\begin{array}{l}\text { Mayor generación y uso de información climática en la toma de } \\
\text { decisiones (sistemas de alerta temprana) }\end{array}$ \\
\hline $\begin{array}{l}\text { Conservación de bosques y aumento de las reservas forestales } \\
\text { de carbono y su capacidad de captura }\end{array}$ & Conservación de ecosistemas y gestión de la diversidad forestal \\
\hline \multicolumn{2}{|c|}{ Indicadores de impacto } \\
\hline $\begin{array}{l}\text { Reducción de emisiones durante toda la vida del proyecto (en } \\
\left.\text { toneladas de } \mathrm{CO}_{2}\right) \text {. Incremeto de capturas de carbono }\end{array}$ & $\begin{array}{l}\text { Cambios esperados en la pérdida de vidas, el valor de los } \\
\text { activos físicos, los medios de subsistencia y/o las pérdidas } \\
\text { ambientales o sociales debido a los efectos del cambio climático }\end{array}$ \\
\hline
\end{tabular}




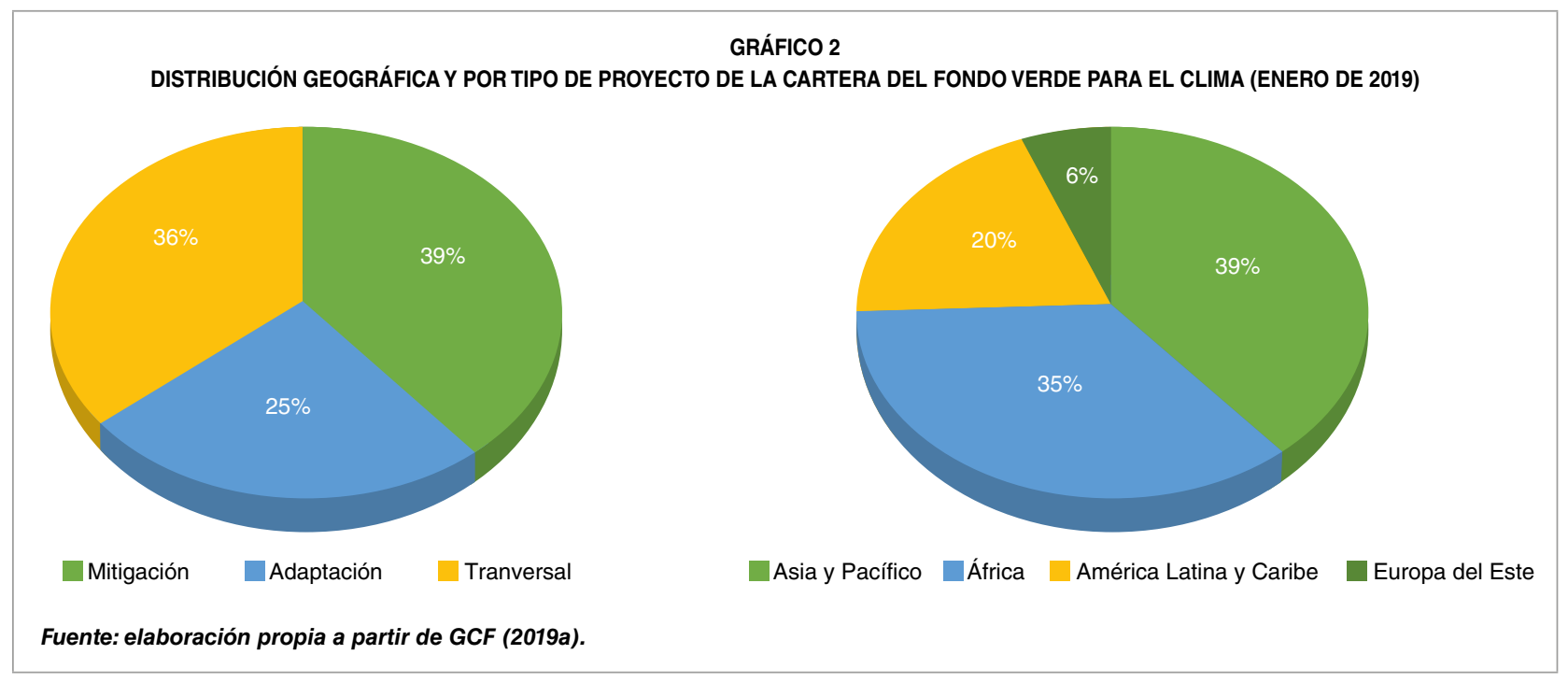

de impacto. Asimismo, el $47 \%$ de los recursos aportados por el fondo a la cartera actual de proyectos ha sido en términos de donación, mientras que el $42 \%$ ha sido en forma de financiación reembolsable vía préstamos. El peso de los instrumentos de capital y garantías es menor ( $9 \%$ y $2 \%$, respectivamente). Desde un punto de vista geográfico, como se observa en el Gráfico 2, la cartera actual del Fondo Verde está principalmente orientada a las regiones de Asia-Pacífico (39\%) y África (35\%), mientras que América Latina y el Caribe representan un $20 \%$ y Europa del Este, un 6\%. Actualmente se está trabajando en una cartera de potenciales proyectos por importe total de 17.000 millones de dólares (GCF, 2019a).

\section{Gobernanza y contribuciones}

El Fondo Verde para el Clima tiene su sede en Songdo (Corea del Sur) y las decisiones se toman a través de un Consejo (Board) que está constituido por los 194 países firmantes de la Convención Marco de Naciones Unidas sobre el Cambio Climático, que están representados a través de 24 «sillas» (12 procedentes de países en desarrollo y otros 12 de países desarrollados ${ }^{7}$ ).

Actualmente, los compromisos totales de los contribuyentes al Fondo alcanzan los 10.300 millones de dólares, e incluyen a 43 países, tres regiones (todas ellas en Bélgica) y una ciudad (París). A día de hoy, se ha desembolsado cerca del $70 \%$ de dichos compromisos y se está trabajando en la primera reposición de capital. En este punto destaca la incertidumbre generada por el anuncio de Estados Unidos, en junio de 2017, de no atender sus compromisos de capital, ratificados en 2014 por la Administración Obama ${ }^{8}$. Por su parte, España ha comprometido $120 \mathrm{mi}-$ llones de euros, de los que ya ha desembolsado 42 millones, situándose en la undécima posición como donante y la decimosexta por importe desembolsado (Banco Mundial, 2019) (Gráfico 3).

\footnotetext{
7 España participa en el Consejo desde su constitución, compartiendo silla con Italia.

8 Estados Unidos se había comprometido a aportar al Fondo 3.000 millones de dólares, si bien por el momento solo ha desembolsado 1.000 millones.
} 


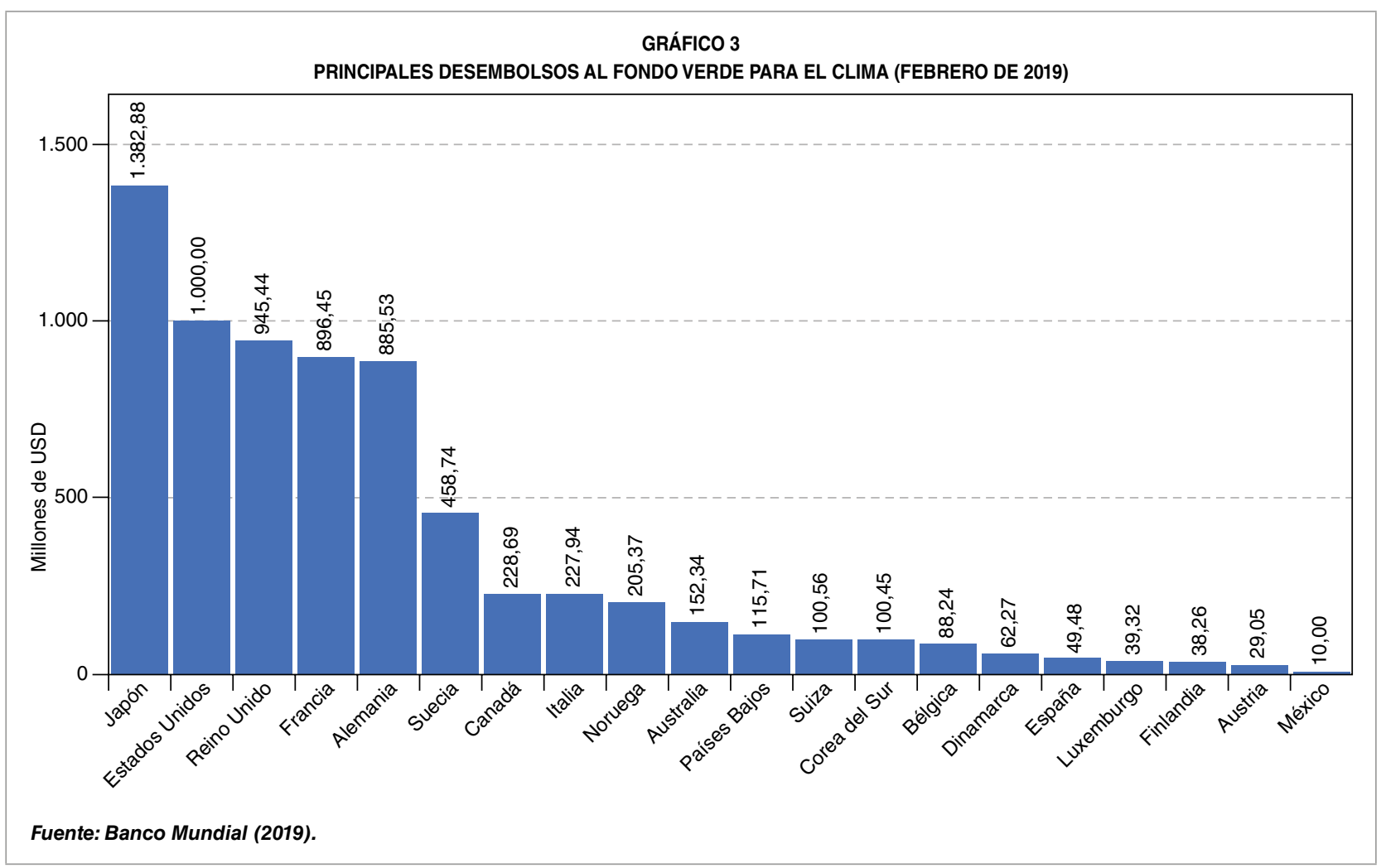

\section{Entidades acreditadas}

El Fondo Verde para el Clima no implementa los proyectos directamente, sino a través de dos figuras importantes: las «Autoridades $\mathrm{Na}$ cionales Designadas» (NDAs, por sus siglas en inglés) y las entidades acreditadas. Las NDAs son instituciones locales de cada país elegible para el Fondo Verde que tienen como función garantizar que los proyectos y programas financiados por este sean coherentes con las normas y políticas nacionales. Es por ello que cada proyecto presentado al Fondo Verde para su aprobación debe contar con la no objeción de la NDA del país o países donde se va a ejecutar (GCF, 2018b).

Por su parte, las entidades acreditadas son aquellas instituciones y organismos que han superado un exhaustivo proceso de homologación de sus procedimientos internos, de tal forma que pueden gestionar los recursos del
Fondo Verde, al mismo tiempo que se comprometen a estructurar la operación (aportando financiación y asumiendo parte del riesgo de la misma), supervisar todo el proceso de ejecución e implementación del proyecto y ser el responsable último del éxito del mismo. Actualmente existen 75 entidades acreditadas, de las cuales casi la mitad (el $45 \%$ ) son de carácter internacional, destacando desde agencias de Naciones Unidas (como la Organización de Naciones Unidas para la Alimentación y la Agricultura - FAO-, el Fondo Internacional de Desarrollo Agrícola -FIDA-, el Programa de Naciones Unidas para el Desarrollo -PNUD—, o el Programa de Naciones Unidas para el Medio Ambiente) hasta grandes bancos multilaterales de desarrollo (BAfD, BaD, BEI, BERD, BID y BID Invest, Banco Mundial, Corporación Financiera Internacional - IFC-), agencias y bancos de desarrollo nacionales (como las francesas AFD y PROPARCO, $D$ 
las alemanas $\mathrm{KfW}$ y $\mathrm{GIZ}$, la holandesa FMO, la austriaca ADA y la japonesa JICA) o bancos privados (como BNP Paribas, Crédite Agricole, Deutsche Bank o HSBC), entre otros (GCF, 2019b).

Actualmente, en España solo una entidad ha superado el riguroso proceso de acreditación: se trata de COFIDES. Esta compañía cuenta con treinta años de experiencia en la financiación de proyectos privados a nivel internacional, buscando un enfoque de impacto en desarrollo. La acreditación habilita a COFIDES a presentar al Fondo Verde para el Clima potenciales proyectos de mitigación y adaptación tanto de carácter público como privado. La compañía tiene amplia experiencia en estos sectores, habiendo financiado en los últimos siete años más de 35 proyectos privados de mitigación y adaptación por importe superior a los 180 millones de euros (lo que ha permitido movilizar una inversión total cercana a los 5.500 millones de euros) (Curbelo y Madrazo, 2018), gracias a su amplia gama de instrumentos, que van desde el capital (capital interés, acotado o a precio abierto) hasta el préstamo (tanto sénior como subordinado).

La participación activa de COFIDES en uno de los principales mecanismos de financiación climática multilateral abre un importante abanico de posibilidades a las empresas para apoyar acciones climáticas en los países en desarrollo y, al mismo tiempo, ayudará a la consecución de los compromisos españoles a nivel internacional.

\section{Conclusión}

Según el Informe Stern (Stern, 2006), el coste de la inacción ante los riesgos globales del cambio climático llegará a suponer entre el $5 \%$ y el $20 \%$ anual del PIB mundial, cantidad muy superior al coste estimado de tomar las acciones necesarias para reducir las emisiones de gases de efecto invernadero, valorado en un $1 \%$ anual del PIB mundial.

El Acuerdo de París abre por primera vez la puerta a buscar soluciones conjuntas y globales al grave problema del sobrecalentamiento global. Existe consenso a nivel internacional en que para la consecución de un desarrollo de bajas emisiones y resiliente al clima se necesita una potente arquitectura financiera climática a nivel global que involucre no solo al sector público, incluyendo los bancos multilaterales y las instituciones financieras de desarrollo, sino también al sector privado.

No obstante, la consecución de los objetivos del Acuerdo de París está sujeta a importantes retos de muy diversa índole (financieros, regulatorios, de concienciación e involucración, etcétera). En lo que se refiere a los retos de la financiación climática se destacan los siguientes. En primer lugar, crear una arquitectura financiera climática lo suficientemente ágil y dinámica, evitando solapamientos e ineficiencias que pueden generarse por la multiplicidad de actores. En segundo lugar, superar las incertidumbres generadas por el anuncio de Estados Unidos de no cumplir con sus compromisos de financiación, lo que mermaría el objetivo de alcanzar los 100.000 millones de dólares anuales para 2020. En tercer lugar, conseguir involucrar al sector privado no solo como financiador, sino también como actor relevante en la consecución de una economía neutra en carbono y alejada de los combustibles fósiles. Para conseguir este importante reto, el papel del sector público es esencial, tanto como catalizador de la iniciativa privada como para poner en marcha políticas fiscales, sociales y de solidaridad que hagan más armoniosa la transición. 


\section{EL PAPEL DE LA FINANCIACIÓN CLIMÁTICA EN LA CONSECUCIÓN DEL ACUERDO DE PARÍS}

\section{Bibliografía}

[1] Acclimatise (2017). Green Climate Fund Proposal Toolkit 2017: Toolkit to develop a project proposal for the GCF. Recuperado de https:// cdkn.org/wp-content/uploads/2017/06/GCFproject-development-manual.pdf

[2] Banco Mundial (2015). From billions to triIlions: $M D B$ contributions to financing for development. Washington, D.C. World Bank Group.Recuperado dehttp://documents.worldbank.org/curated/en/602761467999349576/ From-billions-to-trillions-MDB-contributionsto-financing-for-development

[3] Banco Mundial (2019). Green Climate Fund total contributions. Recuperado de http://fiftrustee.worldbank.org/Pages/gcf.aspx

[4] BERD (2018). Joint reporton multilateral development banks' climate finance 2017. BERD. Londres, junio de 2018. Recuperado de www.ebrd. com/2017-joint-report-on-mdbs-climate-finance

[5] Bird, N., Watson, C., y Schalatek, L. (2017). The Global Climate Finance Architecture. Climate Funds Update. Washington DC: Overseas Development Institute, Heinrich Böll Stiftung North America.

[6] Burchardt, J., Gerbert, P., Schönberger, S., Herhold, P., y Brognaux, C. (2018). The Economic Case for Combating Climate Change. BCG Henderson Institute, septiembre de 2018. Recuperado de https://www.bcg.com/ publications/2018/economic-case-combating-climate-change.aspx

[7] Comisión Europea (2018). Sustainable finance: Commission's Action Plan for a greener and cleaner economy. Nota de prensa del 8 de marzo de 2018. Recuperado de http://europa.eu/rapid/press-release_IP-18-1404_en.htm

[8] Comisión Europea (2019). International Climate Finance. Recuperado de https://ec.europa. eu/clima/policies/international/finance_en

[9] Curbelo, J. L., y Madrazo, R. (2018, 19 de diciembre). Por un desarrollo más sostenible: la oportunidad del Fondo Verde para el Clima. El Economista, Recuperado de https://www.cofides.es/sites/default/files/adjuntos/2018-12 /19.12.18\%20Art\%C3\%ADculo\%20Fondo \%20Verde\%20JLC\%20RM\%20EL\%20 Economista\%202.pdf
[10] European Development Finance Institutions (2017). EDFI Comparative Analysis 2017 (mimeo).

[11] Fabius, L. (2019, 15 de enero). El reto de la financiación sostenible. En la Conferencia Magistral organizada por COTEC y Obra Social La Caixa.

[12] Green Climate Fund (2017). The Green Climate Fund's Private Sector Facility. Recuperado de https://www.greenclimate.fund/documents/20182/194568/The_Green_Climate_ Fund_s_Private_Sector_Facility.pdf/ c47eacd1-5b93-4fe0-97de-b4b9ebe669d3

[13] Green Climate Fund (2018a, diciembre). GCF Handbook. Decisions, policies, and frameworks. Recuperado de https://www.greenclimate.fund/documents/20182/296788/GCF_ Handbook__Decisions__Policies_and_ Frameworks__updated_December_2018_. pdf/25fd22ec-4f81-44ee-b5d1-20bceb2c9264

[14] Green Climate Fund (2018b). GCF Concept Note. User Guide. Recuperado de https://www. greenclimate.fund/documents/20182/239759/ GCF_Concept_Note_User_s_Guide.pdf/ 64866eea-3437-4007-a0e4-01b60e6e463b

[15] Green Climate Fund (2019a). Composición de la cartera actual del Fondo Verde para el Clima. Recuperado de https://www.greenclimate. fund/what-we-do/portfolio-dashboard

[16] Green Climate Fund (2019b). Directorio de entidades acreditadas ante el Fondo Verde para el Clima. Recuperado de https://www. greenclimate.fund/how-we-work/tools/ entity-directory

[17] Ministerio de Economía y Empresa (2019, febrero). Información relativa a los compromisos españoles en materia de financiación climática. España. Recuperado de http://www. mineco.gob.es/portal/site/mineco/menuitem. 32ac44f94b634f76faf2b910026041a0/?vgnextoid $=341295 \mathrm{c} 4 \mathrm{e} 862 \mathrm{c} 310 \mathrm{VgnVCM} 100000$ 2006140aRCRD

[18] Montalvo, A. (2011). La financiación internacional del cambio climático y las negociaciones multilaterales. Revista ICE, (862).

[19] Naciones Unidas (2015). The Paris Agreement. Recuperado de https://unfccc.int/sites/default/ files/english_paris_agreement.pdf

[20] Organización para la Cooperación y el Desarrollo Económico (2017). Invertir en el clima, $\triangleright$ 
Compañía Española de Financiación del Desarrollo (COFIDES)

invertir en el crecimiento. Recuperado de https://www.oecd.org/environment/cc/g20-climate/una-sintesis-investing-in-climate-investing-in-growth.pdf

[21] Programa de Naciones Unidas para el Desarrollo (2018). Concrete outcomes from UN climate change talks in Poland mean it is time to get to work. Recuperado de http://www. undp.org/content/undp/en/home/news-centre/ news/2018/exceeding-expectations-in-katowice.html

[22] Stern, N. (2006, 19 de enero). Stern Review on the Economics of Climate Change. HM Treasury. Recuperado de www.sternreview.org.uk 